\title{
Computational Study on the Boundary Between the Concerted and Stepwise Mechanism of Bimolecular $\mathrm{S}_{\mathrm{N}}$ Ar Reactions
}

\author{
Simon Rohrbach, John A. Murphy, ${ }^{*}$ and Tell Tuttle* \\ Cite This: https://dx.doi.org/10.1021/jacs.0c01975 \\ Read Online
}

\begin{abstract}
The text-book mechanism of bimolecular nucleophilic aromatic substitutions $\left(S_{N} A r\right)$ reactions is a stepwise process that proceeds via a so-called Meisenheimer intermediate. Only recently the alternative, concerted version of this mechanism has gained acceptance as more and more examples thereof have been reported. But so far only isolated examples of concerted $S_{N} A r$ reactions have been described and a coherent picture of when a $\mathrm{S}_{\mathrm{N}} \mathrm{Ar}$ reaction proceeds via a stepwise and when via a concerted mechanism has not yet been established. Here key factors are identified that influence the mechanistic choice of $S_{N} A r$ reactions. Moreover, the electron affinity is used as a simple descriptor to make a prediction on whether a given aryl fluoride substrate favors a concerted or stepwise mechanism.
\end{abstract}

\section{INTRODUCTION}

Nucleophilic substitutions on aromatic systems can proceed via several different reaction mechanisms. ${ }^{1}$ Of these, the most fundamental is the bimolecular nucleophilic aromatic substitution $\left(S_{N} A r\right)$ as described in the landmark review by Bunnett et al. ${ }^{2}$ As detailed more recently, ${ }^{3}$ the widely accepted stepwise energy profile of the $S_{N} A r$ reaction appears not to be the only possible route. In fact, over the last decades, more and more reports accumulated in the literature that suggested certain $S_{N} A r$ reactions to follow a concerted pathway. In particular the recent study by Jacobsen et al. was important in raising the general awareness of the two mechanistic domains accessible to $\mathrm{S}_{\mathrm{N}} \mathrm{Ar}$ reactions. ${ }^{4}$ The combined impact of these investigations has now reached a critical momentum. It seems appropriate to fundamentally question the long-established mechanistic picture of the $S_{N}$ Ar reaction.

Probing the mechanism of the $S_{N} A r$ reaction is challenging, however. Experimentally, complex structure-activity relationship studies need to be performed. This approach has been followed by Williams et al. in several thorough studies. ${ }^{5-10}$ Although the concept behind these studies is elegant and intuitive, the approach is limited to certain suitable model systems. With computational tools, in contrast, it is straightforward to determine the energy profile of any imaginable $S_{N} A r$ reaction. Consequently, it is not surprising that most claims for concerted $S_{N} A r$ reactions are based on computational models, including the above-mentioned study by Jacobsen. However, the question arises whether the computational methods used are reliable. Only rarely was a thorough validity of the method established and if so, only for selected examples. ${ }^{11}$ Importantly, it has never been thoroughly

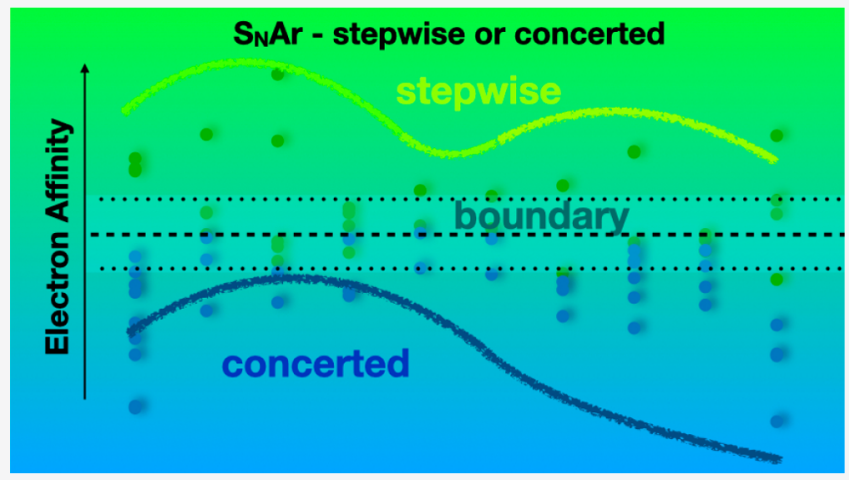

established that a computational model is able to predict correctly the mechanism of both concerted and stepwise $S_{N} A r$ reactions.

Because of these challenges, the exact nature of the $S_{N} A r$ mechanism has only been investigated for isolated examples. Besides some general trends and rough guidelines, there is no coherent picture of when bimolecular $S_{N} A r$ reactions follow a stepwise and when a concerted mechanism. The aim of this work was to first establish the validity of a DFT method to predict the reaction mechanism and then to use this method to investigate the trends of what types of $S_{N} A r$ reactions follow which mechanistic pathway.

For this work to be as beneficial as possible for the community of chemists, it was important to choose appropriate descriptors to parametrize $S_{N} A r$ reactions. Broadly speaking, one expects an $S_{N} A r$ reaction to depend on several components: the nucleophile (and a countercation in case of charged nucleophiles), the leaving group, the aromatic system, and the solvent. There are numerous parameters that would allow any of these three aspects to be described. For the description of the aromatic system, a particularly convenient measure is the Hammett substituent constants. In fact, Hammett correlations have been applied to $S_{N} A r$ reactions

Received: February 19, 2020

Published: August 7, 2020 
in many instances and, usually, a good linear regression was observed for the $\sigma_{\mathrm{p}}{ }^{-}$substituent constant. ${ }^{12,13}$ More recently, the slope of Hammett correlations has also been interpreted as an indication of the mechanistic nature of $S_{N}$ Ar reactions. ${ }^{14-16}$ However, while intuitive, this approach lacks a sound theoretical foundation, as with the exception of particular classes of reactions such as proton-coupled electron transfers, ${ }^{17,18}$ the relationship between the reaction free energy and the mechanism is not explicit-indeed, in the current work there is no observed relationship (see Supporting Information, SI, Figures S15-S18). Moreover, there are no well-established reference values for typical concerted and typical stepwise reactions and often the fact that the Hammett slope is temperature-dependent is ignored. Therefore, instead of analyzing the slope of the linear regression, in this study it was examined whether there is a sharp turning point from a stepwise to a concerted mechanism as the aromatic systems become more electron-rich (i.e., the ring substituents become less electron-withdrawing-this corresponds to a less positive value of the $\sigma_{\mathrm{p}}{ }^{-}$substituent constant). Encouragingly, one isolated example of such a mechanistic turning point on the $\sigma_{\mathrm{p}}{ }^{-}$scale has previously been reported. ${ }^{19}$ The values of the $\sigma_{\mathrm{p}}{ }^{-}$ constants used here were taken from the review by Hansch et al. $^{20}$

Due to the historic importance of Hammett correlations to characterize $S_{N} A r$ reactions and given the fact that generally good correlations have been found, we sought to describe the mechanistic turning point based on the $\sigma_{\mathrm{p}}{ }^{-}$substituent constants. To do so, one can think of a hypothetical parasubstituent for which the energy profile of the $S_{N} A r$ reaction passes through a saddle point (i.e., the curvature and the slope along the reaction coordinate are zero). This substituent would mark the mechanistic turning point; thus, we will refer to its Hammett constant as $\tau_{\mathrm{p}}^{-}$. Any substituent that was more electron-withdrawing would cause the reaction to proceed via a stepwise energy-profile with a local minimum (and a general inflection point-with slope $\neq$ zero-instead of the saddle point), whereas the reaction would proceed via a concerted mechanism for substrates with a less electron-withdrawing substituent.

Since the Hammett ${\sigma_{\mathrm{p}}}^{-}$scale consists of discrete values, a way to approximate $\tau_{p}^{-}$based on the chemically existing and viable para-substituents was needed. This can be achieved by taking the least electron-withdrawing substituent for which the reaction still follows a stepwise energy profile $\left(\sigma_{\mathrm{p}, \text { min-stepwise }}^{-}\right)$ and the most electron-withdrawing substituent for which the reaction still follows a concerted energy profile $\left(\sigma_{\mathrm{p}, \max -\text { concerted }}^{-}\right)$. Then the average of the two substituent constants is calculated (eq 1). This value gives a measure for how sharply the mechanistic turning point is projected onto the Hammett $\sigma_{\mathrm{p}}{ }^{-}$ scale. In other words, $\Delta \sigma_{p}^{-}$shows how precisely the mechanistic turning point can be possibly known based on the available $\sigma_{\mathrm{p}}{ }^{-}$values.

$$
\Delta \sigma_{\mathrm{p}}^{-}=\frac{\sigma_{\mathrm{p}, \text { min_stepwise }}^{-}-\sigma_{\mathrm{p}, \text { max_concerted }}^{-}}{2}
$$

The turning point itself can then be expressed according to eq 2.

$$
\tau_{\mathrm{p}}^{-}=\sigma_{\mathrm{p}, \text { max_concerted }}^{-}+\Delta \sigma_{\mathrm{p}}^{-}
$$

\section{RESULTS AND DISCUSSION}

A thorough benchmarking study showed that the M11 functional is well suited to predict the mechanism of $S_{N} A r$ reactions. (Full details of the functional evaluation study and further validation studies for the M11 functional can be found in the SI).

To gain a broad overview of the two mechanistic domainsstepwise vs concerted $S_{N} A r$ - three classes of $S_{N} A r$ reactions were investigated. These are the halide displacement with potassium methoxide (Figure 1), halide-halide exchange reactions (Tables S2-4) and the analogous chalcogenchalcogen exchange reactions (Tables $\mathrm{S} 2-5$ ).

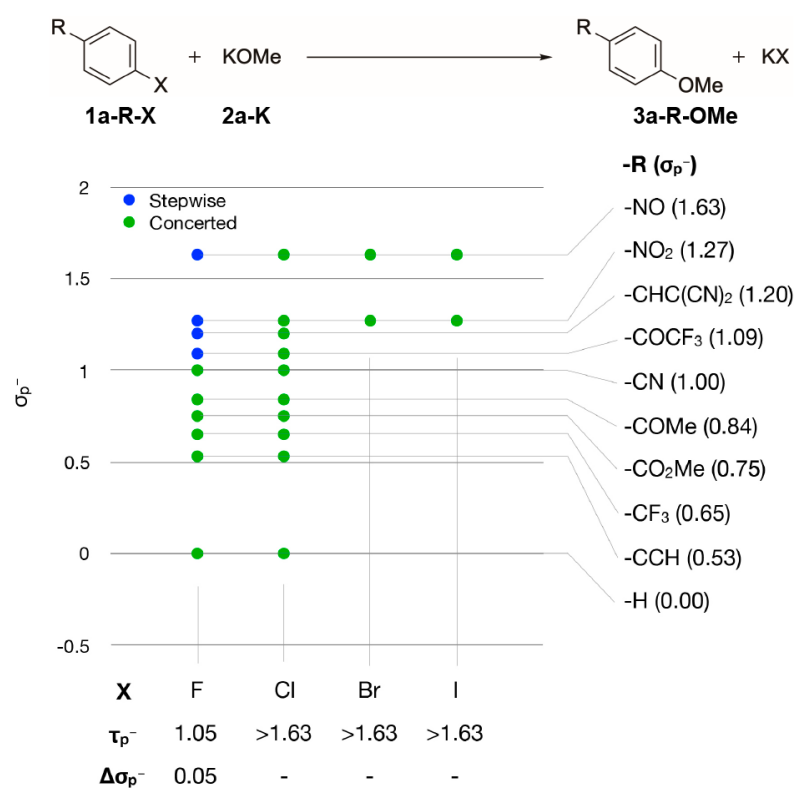

Figure 1. Mechanistic turning point for the halide displacement by potassium methoxide and benzene derivatives. A stepwise mechanism was only observed for examples with fluoride as a leaving group and strongly electron-withdrawing substituents.

Only for the fluoride series was the mechanistic turning point identified, with $\tau_{\mathrm{p}}{ }^{-}=1.05$ (Figure 1). For the displacement of chloride, bromide, and iodide the mechanistic turning point does not fall into the chemically plausible range of the $\sigma_{\mathrm{p}}{ }^{-}$scale. In fact, these reactions all showed a concerted energy profile even with the most electron-withdrawing paranitroso substituent that was considered in this study.

A similar picture was obtained for the halide exchange reactions (Tables S2-S4). The mechanistic turning point for most of the chalcogen exchange reactions, in contrast, actually fell mainly onto the applied $\sigma_{\mathrm{p}}{ }^{-}$scale (Tables S2-S5). In general, a concerted mechanism is favored for the chalcogen exchange reaction by the participation of larger (i.e., softer) chalcogens. The analogous statement holds true for the halide exchange reaction. Overall, the halides chloride, bromide and iodide all strongly favor a concerted mechanism, either in the halide exchange reaction or in an exchange reaction with potassium methoxide (Figure 1). Only for the $S_{N} A r$ reactions involving fluoride a stepwise energy profile was found to have significant importance. Because of this finding and because fluoride is the prototype leaving group for $S_{N} A r$ reactions, further discussion will focus on $S_{N} A r$ displacements of fluoride. 
So far, only the potassium cation has been considered as a countercation in the examined model systems. The effect of the countercation on the $S_{N} A r$ mechanism was studied, based on the displacement of fluoride from 1a-R-F with different alkali metal methoxide salts 2a-M (Figure 2). As a general

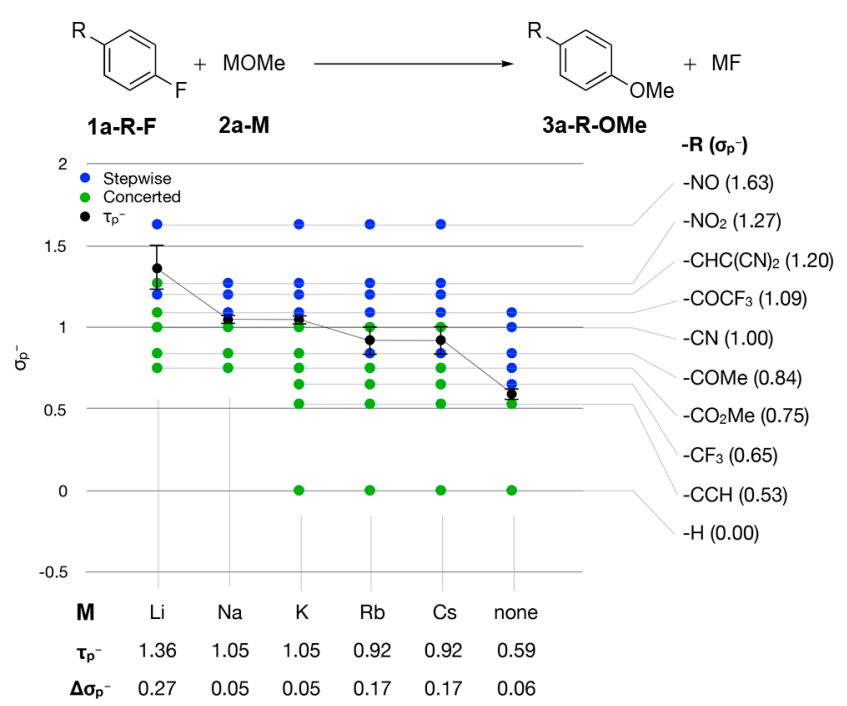

Figure 2. As the countercation gets larger, the reaction tends to favor a stepwise mechanism.

trend, the stepwise mechanism becomes more dominant with increasing size of the countercation, i.e., the value for $\tau_{\mathrm{p}}{ }^{-}$ decreases as the countercation becomes larger. This trend culminates in the extreme case where no countercation is present at all. Closer inspection of the data shows that there is no or only a small difference between the reactions with the cations sodium, potassium, rubidium, and cesium. Only the boundary cases with lithium as a countercation on the one hand and without a countercation at all on the other hand show a pronounced change of the $\tau_{\mathrm{p}}{ }^{-}$value.

The observed trend suggests that the better the countercation can stabilize the negative charge on the fluoride leaving group, the more strongly a concerted reaction mechanism is favored. This is in agreement with the trend of decreasing lattice energy of alkali fluoride salts with increasing atomic number of the alkali cation (i.e., weaker bonding between the fluoride anion and the alkali metal cation). ${ }^{21}$ The observed trend is also in line with observations made on the computational investigation $^{20}$ of the amide $\alpha$-arylation by Clayden et al. $^{14}$ (Including explicit solvent molecules in the model only had a minor effect on the $\tau_{\mathrm{p}}{ }^{-}$value, see the SI).

Keeping the fluoride leaving group, the potassium cation and the aromatic core constant, the mechanistic turning point was investigated for different nucleophiles (Figure 3). It was found that potassium methanethiolate $\mathbf{2 b}-\mathbf{K}$, potassium azide $2 \mathbf{c}-\mathbf{K}$, and the two carbon nucleophiles $\mathbf{2 d - K}$ and $\mathbf{2 f - K}$ all have the same mechanistic turning point $\tau_{\mathrm{p}}{ }^{-}$as potassium methoxide. The $S_{N} A r$ reaction of the nucleophile $2 e-K$ is the only exception.

This series of reactions favors a concerted mechanism more $\left(\tau_{\mathrm{p}}{ }^{-}=1.36\right)$ than the other investigated reaction series $\left(\tau_{\mathrm{p}}{ }^{-}=\right.$ 1.05). Closer inspection of the geometries of the rate limiting transition states with nucleophile $2 \mathbf{e}-\mathbf{K}$ showed that steric repulsion (i.e., dispersion interactions) may be at the heart of this pronounced tendency to follow a concerted mechanism

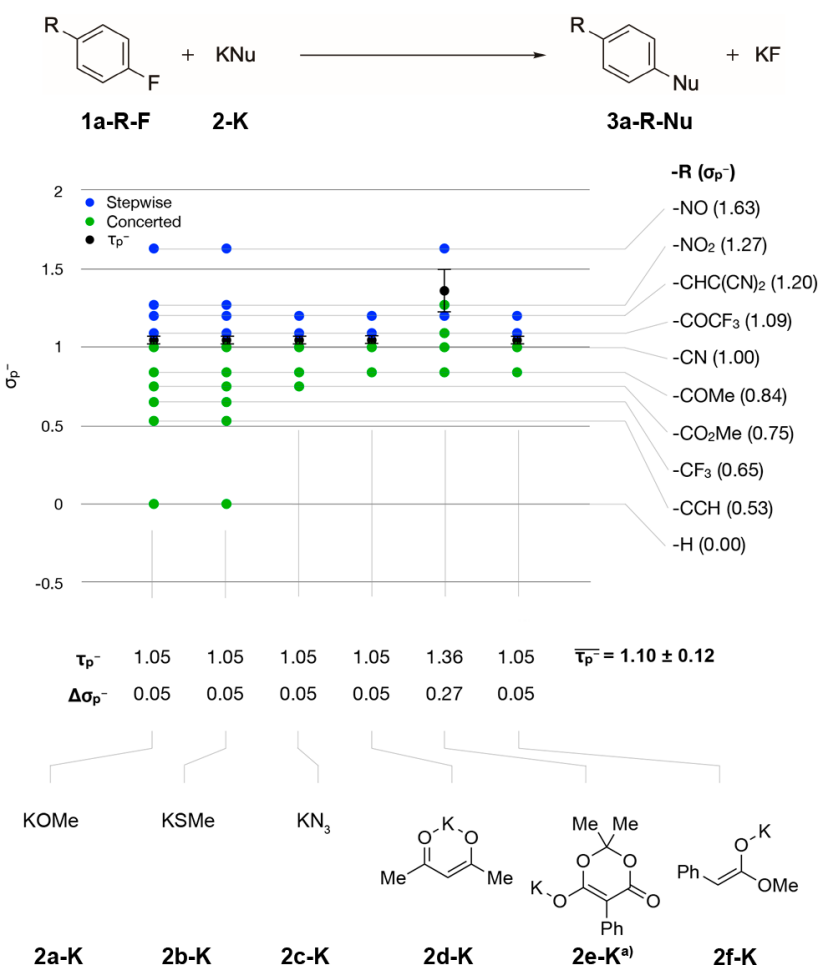

Figure 3. A similar mechanistic turning point was found for a number of very different nucleophiles. With $\mathrm{R}=\mathrm{NO}$ the reaction likely follows a $S_{N}(E T) A r$ pathway (see Supporting Information).

(see Figure S5, see Figure S8 further discussion of such effects). The average $\overline{\tau_{\mathrm{p}}^{-}}$over all six nucleophiles was $1.10 \pm$ 0.12 for the substrate series $\mathbf{1 a}-\mathbf{R}-\mathbf{F}$.

The observation that a number of very different nucleophiles showed the same mechanistic turning point was somewhat surprising. The same investigation for an additional two aromatic systems-pyridine and naphthalene-led to a similar conclusion (see the SI).

Since there is relatively little variation between different nucleophiles attacking the same aromatic substrate, (i.e., the value $\tau_{\mathrm{p}}{ }^{-}$is mainly characteristic for the aromatic system and the fluoride leaving group), it is, in principle, sufficient to examine the mechanistic turning point with one nucleophile only in order to characterize the mechanistic preference of a given aryl fluoride electrophile. Hence, potassium methoxide was selected as the probe nucleophile and the aromatic systems 1a-R-F to 1e-R-F (Figure 4) were investigated. It can be seen that a stepwise reaction profile became more favored as either the aromatic system was extended (going from $\mathbf{1 a - R - F}$ to $\mathbf{1 b}$ R-F to 1c-R-F) or nitrogen atoms were introduced (going from 1a-R-F to $\mathbf{1 d - R - F}$ to $1 \mathbf{e}-\mathrm{R}-\mathrm{F})$. The effect of one additional fused benzene ring equaled approximately the effect of one additional nitrogen atom. The observed trends have an intuitive explanation. An additional fused ring, or a nitrogen atom in the ring, help to stabilize the negative charge that accumulates on the aromatic system during the addition of the nucleophile. The better the aromatic core is able to accommodate this negative charge, the less the stabilization of a (potential) Meisenheimer intermediate depends on the electron-withdrawing nature of the para-substituent.

Obviously, the mechanistic turning point is highly sensitive to the nature of the aromatic system beyond the parasubstituent. Hence, the next question is whether a readily 


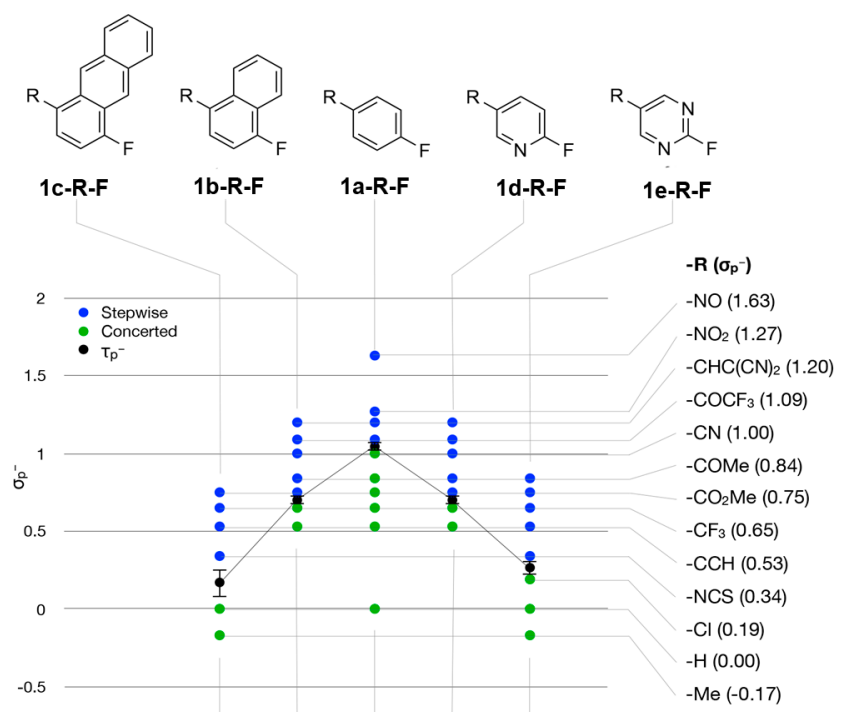

Aromatic Core

$$
\begin{array}{rrrrrr}
\tau_{\mathrm{p}^{-}} & 0.17 & 0.70 & 1.05 & 0.70 & 0.27 \\
\Delta \sigma_{\mathrm{P}^{-}} & 0.17 & 0.05 & 0.05 & 0.05 & 0.08
\end{array}
$$

Figure 4. The better the aromatic core can accommodate the accumulating negative charge in the transition state, the more strongly a stepwise mechanism is favored.

accessible descriptor can be identified that allows to classify aryl fluorides according to what $S_{\mathrm{N}} \mathrm{Ar}$ mechanism they are likely to follow. The calculated gas-phase electron affinity was found to be a suitable measure to do this (Figure 5). (Other descriptors have been considered as well but were found inferior, see SI). Analogous to $\tau_{\mathrm{p}}{ }^{-}$, we calculated the turning

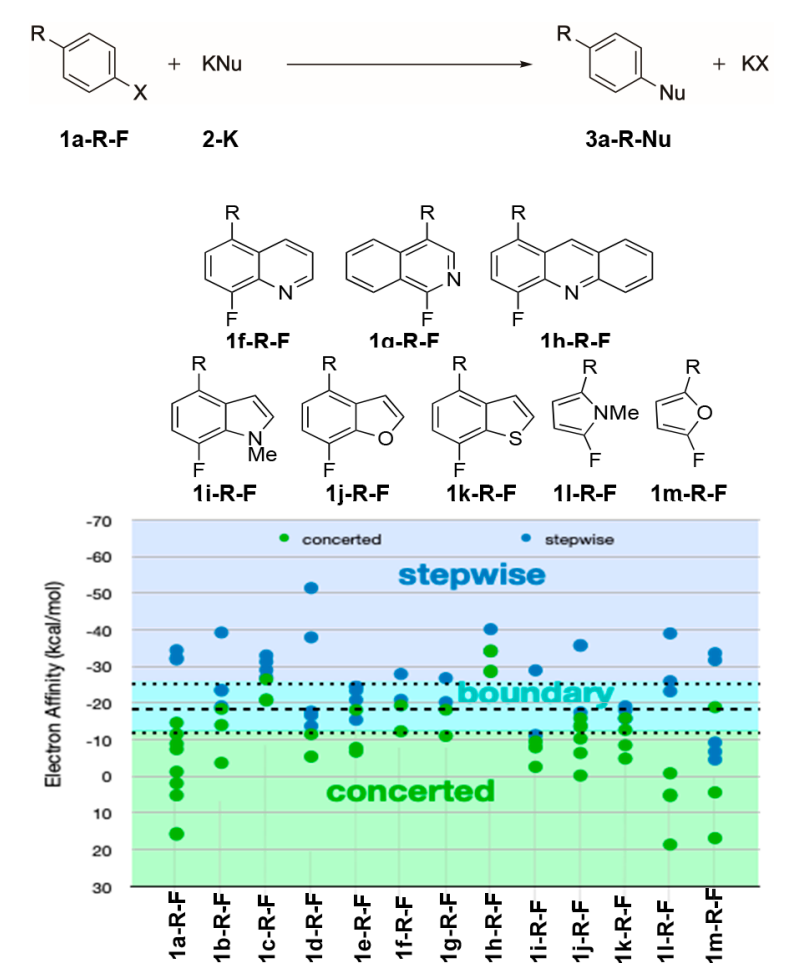

Figure 5. Electron affinity of an aryl fluoride compound allows for a quick classification of what $S_{N} A r$ mechanism the substrate in question is likely to follow. The dashed (---) line indicates the average over all $\tau_{\mathrm{p}}{ }^{-}$and the dotted lines $(\cdots)$ mark the standard deviation. point electron affinity $\mathrm{EA}_{\mathrm{T}}$ according to eq 3 where $\mathrm{EA}_{\text {max stepwise }}$ is the largest electron affinity for which the reaction proceeds stepwise, whereas $\mathrm{EA}_{\text {min concerted }}$ is the smallest electron affinity for which the reaction proceeds in a concerted manner.

Note that the sign of the electron affinity axis is opposite to the sign of the Hammett substitution constant axis.

$$
\mathrm{EA}_{\mathrm{T}}=\mathrm{EA}_{\text {max_stepwise }}+\frac{\mathrm{EA}_{\text {min_concerted }}-\mathrm{EA}_{\text {max_stepwise }}}{2}
$$

In Figure 5, the mechanistic pathway and the electron affinity of the substrate is shown for 14 series of aromatic system. The average $\mathrm{EA}_{\mathrm{T}}$ over these 14 examples was found to be $(-18.3 \pm 6.7) \mathrm{kcal} / \mathrm{mol}$. Thus, if an aryl fluoride substrate shows an electron affinity of smaller than $-25.0 \mathrm{kcal} / \mathrm{mol}$, it is likely to undergo a stepwise $S_{N} A r$ reaction, whereas an electron affinity of greater than $-11.6 \mathrm{kcal} / \mathrm{mol}$ indicates the preference for a concerted pathway. We note the exception of the $\mathbf{1 h}-\mathbf{R}-\mathbf{F}$ series from the general trend. This is due to the localization of the frontier orbitals in the $S_{N} A r$ transition state for this series, whereas the SOMO of the corresponding radical anion (used to calculate the EA) is delocalized across the ring system.

As mentioned at the outset, the slope of the Hammett correlation for series of $S_{N} A r$ reactions was often used in the literature as an indicator for the nature of the mechanism. In this work, a different angle is taken on the Hammett correlation, instead. It builds on the fact that the mechanism of the $S_{N} A r$ reaction changes as the para-substituent is varied. This indicates already that the slope of the Hammett correlation is an unsuitable measure for the mechanistic nature of $S_{N} A r$ mechanism. Also, a more detailed analysis of the Hammett correlation showed that there is no signature for the mechanistic turning point in the Hammett correlation (Figure 6 ). For example, the two nucleophiles $2 \mathbf{a}-\mathbf{K}$ and $\mathbf{2 b}-\mathbf{K}$ have the

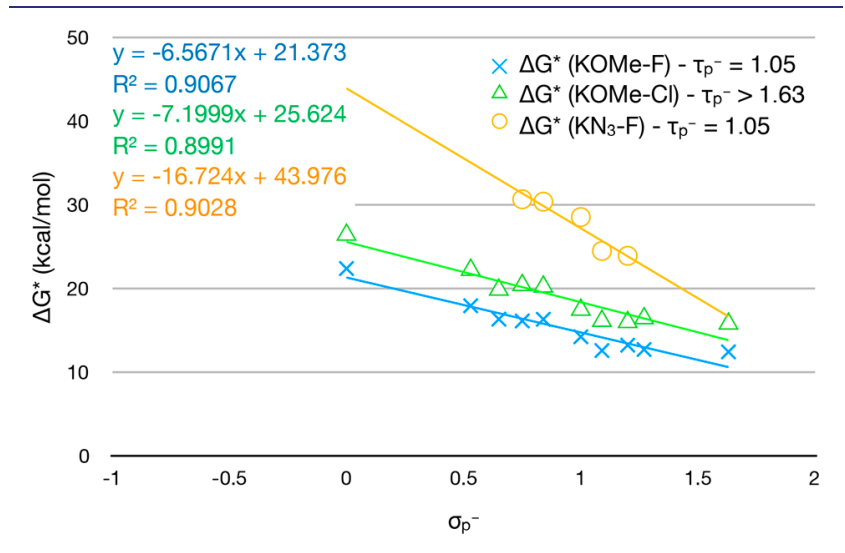

Figure 6. Activation energy of the rate limiting step of a series of $S_{N} A r$ reactions does not contain information about the overall mechanism.

same mechanistic turning point but a different slope in the Hammett correlation when reacting with the electrophile 1a-RF. Alternatively, the electrophiles 1a-R-F and 1a-R-Cl have a similar slope but different turning point when reacting with the nucleophile 2a-K. Further, no kink can be found in the correlation. (Additionally, key geometric properties of the ratelimiting transition state were investigated in an analogous correlation. Again, the correlation did not harbor any information about the mechanistic turning point-see the SI). 
It becomes evident that in general the rate limiting step does not contain information about the nature of the $S_{N} A r$ mechanism. The mechanistic choice depends on the features of the bond that is more easily broken (or formed). In light of this conclusion it becomes obvious why the reaction series of different nucleophiles with the same aromatic system have roughly the same value of $\tau_{\mathrm{p}}{ }^{-}$(Figure 3 ). In all these apparently different reactions, the key step where the nature of the mechanism is decided is the same-the expulsion of the fluoride leaving group.

\section{CONCLUSIONS}

On the basis of a thoroughly bench-marked computational model, an in-depth analysis of factors influencing the choice of the reaction mechanism-concerted or stepwise-has been performed for the $S_{N} A r$ reaction. Besides obvious trends such as a preference for the stepwise mechanism in systems that can accommodate the buildup of negative charge better (e.g., larger aromatic cores or the presence of nitrogen heteroatoms), it was found that the nature of the incoming nucleophile has only a minimal influence on the mechanistic choice. The electron affinity was found to be a readily available descriptor to judge the mechanistic preference of a particular aryl fluoride acting as the electrophile in a $S_{N} A r$ reaction. Finally, it was demonstrated that the slope of the Hammett correlation is a potentially misleading indicator of the $S_{N} A r$ mechanism of a reaction class. Consequently, it should no longer be considered when discussing the mechanism of $S_{N} A r$ reactions. Instead, the electron affinity offers a better alternative indicator.

\section{ASSOCIATED CONTENT}

\section{SI Supporting Information}

The Supporting Information is available free of charge at https://pubs.acs.org/doi/10.1021/jacs.0c01975.

Description and validation of the method, additional examples, and descriptions for every computational output file (PDF)

Data set underlying this research, $10.15129 / 254$ ecae89c72-4bb9-9319-b16eada94f9c

\section{AUTHOR INFORMATION}

\section{Corresponding Authors}

John A. Murphy - Department of Pure and Applied Chemistry, University of Strathclyde, Glasgow G1 1XL, United Kingdom; 이이.org/0000-0003-3136-0845; Email: John.Murphy@ strath.ac.uk

Tell Tuttle - Department of Pure and Applied Chemistry, University of Strathclyde, Glasgow G1 1XL, United Kingdom; ○ orcid.org/0000-0003-2300-8921; Email: tell.tuttle@ strath.ac.uk

\section{Author \\ Simon Rohrbach - Department of Pure and Applied Chemistry, University of Strathclyde, Glasgow G1 1XL, United Kingdom; 응 orcid.org/0000-0002-1007-1553}

Complete contact information is available at:

https://pubs.acs.org/10.1021/jacs.0c01975

\section{Author Contributions}

The manuscript was written through contributions of all authors. All authors have given approval to the final version of the manuscript.

\section{Notes}

The authors declare no competing financial interest.

\section{ACKNOWLEDGMENTS}

Financial support for this work was provided by GSK via the GSK/University of Strathclyde Centre for Doctoral Training in Synthetic and Medicinal Chemistry. Further funding was provided by the EPSRC via Prosperity Partnership EP/ S035990/1, whom we thank. Results were obtained using the ARCHIE-WeSt High Performance Computer (www. archie-west.ac.uk) based at the University of Strathclyde.

\section{ABBREVIATIONS USED}

$S_{\mathrm{N}} \mathrm{Ar}$, nucleophilic aromatic substitution; DFT, density functional theory

\section{REFERENCES}

(1) Terrier, F. Modern Nucleophilic Aromatic Substitutions; WileyVCH: Hoboken, NJ, 2013.

(2) Bunnett, J. F.; Zahler, R. E. Aromatic Nucleophilic Substitution Reactions. Chem. Rev. 1951, 49 (2), 273-412.

(3) Rohrbach, S.; Smith, A. J.; Pang, J. H.; Poole, D. L.; Tuttle, T.; Chiba, S.; Murphy, J. A. Concerted Nucleophilic Aromatic Substitution Reactions. Angew. Chem., Int. Ed. 2019, 58, 1636816388 .

(4) Kwan, E. E.; Zeng, Y.; Besser, H. A.; Jacobsen, E. N. Concerted nucleophilic aromatic substitutions. Nat. Chem. 2018, 10, 917-923.

(5) Renfrew, A. H. M.; Taylor, J. A.; Whitmore, J. M. J.; Williams, A. A single transition state in nucleophilic aromatic substitution: reaction of phenolate ions with 2-(4-nitrophenoxy)-4,6-dimethoxy-1,3,5triazine in aqueous solution. J. Chem. Soc., Perkin Trans. 2 1993, $1703-1704$.

(6) Hunter, A.; Renfrew, M.; Taylor, J. A.; Rettura, D.; Whitmore, J. M. J.; Williams, A. Stepwise versus Concerted Mechanisms at Trigonal Carbon: Transfer of the 1, 3, 5-Triazinyl Group between Aryl Oxide Ions in Aqueous Solution. J. Am. Chem. Soc. 1995, 117, 5484-5491.

(7) Cullum, N. R.; Renfrew, A. H. M.; Rettura, D.; Taylor, J. A.; Whitmore, J. M. J.; Williams, A. Effective Charge on the Nucleophile and Leaving Group during the Stepwise Transfer of the Triazinyl Group between Pyridines in Aqueous Solution. J. Am. Chem. Soc. 1995, 117, 9200-9205.

(8) Shakes, J.; Raymond, C.; Rettura, D.; Williams, A. Concerted displacement mechanisms at trigonal carbon: the aminolysis of 4aryloxy-2,6-dimethoxy-1,3,5-triazines. J. Chem. Soc., Perkin Trans. 2 1996, $1553-1557$.

(9) Cullum, N. R.; Rettura, D.; Whitmore, J. M. J.; Williams, A. The aminolysis and hydrolysis of $\mathrm{N}$-(4,6-diphenoxy-1,3,5-triazin-2-yl) substituted pyridinium salts: concerted displacement mechanism. J. Chem. Soc., Perkin Trans. 2 1996, 1559-1563.

(10) Renfrew, A. H. M.; Taylor, J. A.; Whitmore, J. M. J.; Williams, A. Timing of bonding changes in fundamental reactions in solutions: pyridinolysis of a triazinylpyridinium salt. J. Chem. Soc., Perkin Trans. 2 1994, 2383-2385.

(11) Chéron, N.; Jacquemin, D.; Fleurat-Lessard, P. A qualitative failure of B3LYP for textbook organic reactions. Phys. Chem. Chem. Phys. 2012, 14, 7170-7175.

(12) Miller, J. Electrophilic and nucleophilic substitution in the Benzene ring and the Hammett Equation. Aust. J. Chem. 1956, 9, 6173.

(13) Miller, J.; Kai-Yan, W. The $\mathrm{S} N$ mechanism in aromatic compounds. Part XXIX. Some para-substituted chlorobenzenes. J. Chem. Soc. 1963, 3492.

(14) Leonard, D. J.; Ward, J. W.; Clayden, J. Asymmetric $\alpha$-arylation of amino acids. Nature 2018, 562, 105-109. 
(15) Ong, D. Y.; Tejo, C.; Xu, K.; Hirao, H.; Chiba, S. Hydrodehalogenation of Haloarenes by a Sodium Hydride-Iodide Composite. Angew. Chem., Int. Ed. 2017, 56, 1840-1844.

(16) Handel, H.; Pasquini, M. A.; Pierre, J. L. Effets de cryptands et activation de bases-VII11 Partie précédente Réf. 1. Réduction des halogénures de phényle par l'hydrure de potassium. Tetrahedron 1980, 36, 3205-3208.

(17) Liu, T.; Guo, M.; Orthaber, A.; Lomoth, R.; Lundberg, M.; Ott, S.; Hammarstrom, L. Accelerating proton-coupled electron transfer of metal hydrides in catalyst model reactions. Nat. Chem. 2018, 10, 881887.

(18) Sayfutyarova, E. R.; Lam, Y.-C.; Hammes-Schiffer, S. Strategies for Enhancing the Rate Constant of $\mathrm{C}-\mathrm{H}$ Bond Cleavage by Concerted Proton-Coupled Electron Transfer. J. Am. Chem. Soc. 2019, 141, 15183-15189.

(19) Sun, H.; DiMagno, S. G. Room-temperature nucleophilic aromatic fluorination: experimental and theoretical studies. Angew. Chem., Int. Ed. 2006, 45, 2720-2725.

(20) Hansch, C.; Leo, A.; Taft, R. W. A survey of Hammett substituent constants and resonance and field parameters. Chem. Rev. 1991, 91, 165-195.

(21) Shriver, D.F. Coord. Chem. Rev. 1990, 99, 3. 